\title{
Transversus Abdominis Plane Block: A Scoping Review
}

Liam Maher, BSN; Leanne Orians, BSN; Gabrielle Pappas, BSN; Advisor: Dr. Lanny Coker, DNSc, CRNA College of Nursing - The University of Tennessee Health Science Center - Memphis, TN

\section{Purpose}

The purpose of this project is to provide an overview of the literature on the analgesic effects of transverse abdominal plane (TAP) block and its effectiveness in reducing opioid consumption.

\section{Specific Aims}

- Compare the analgesic effectiveness of TAP block versus opioid-based anesthesia.

- Evaluate postoperative opioid use and pain scores in populations who received a TAP block versus those who did not receive a TAP block.

- Determine the efficacy of the introduction of TAP blocks within a multimodal anesthetic as part of a postoperative pain management plan.

\section{Background}

- Multimodal pain

management is integral to enhanced recovery pathways, ye the most effective pain management

strategies have not

been determined. The

use of nerve blocks as

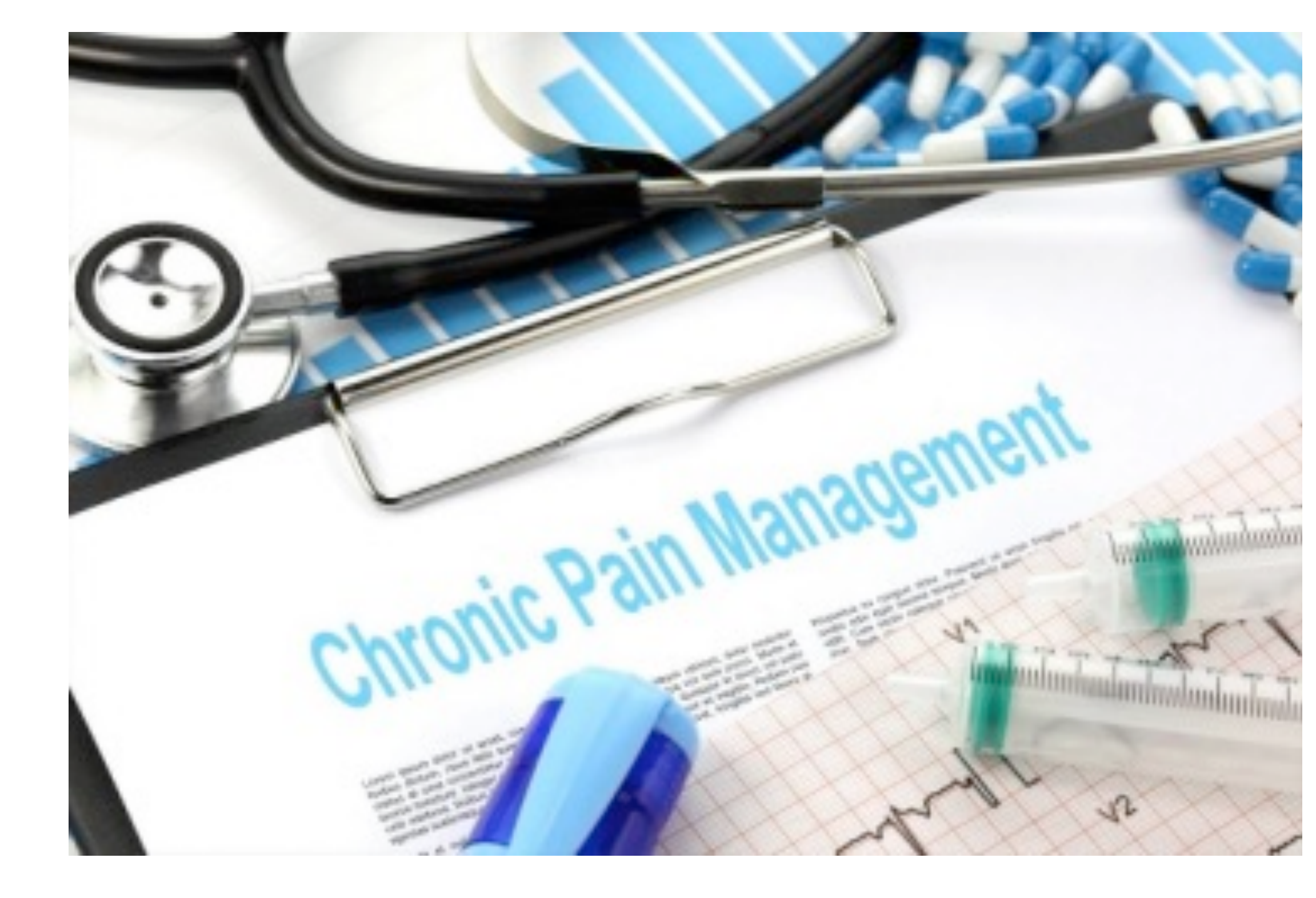

an alternative or integral approach to pain

management in abdominal surgery is an evolving

research topic.

- Pain control during the perioperative period is commonly provided with opioids. However, these analgesic medications have negative aspects, which can be observed in both the perioperative and postoperative periods.

- A key evidence-based practice aspect of the ERAS model is multimodal anesthesia, including the appropriate use of opioids.

- Since the introduction of ultrasound-guided TAP block in 2007, data supporting analgesic efficacy have been conflicting. There is a need to refine and standardize ERAS protocols with focus on pain management and patient satisfaction.

\section{Methods}

Study Design

- Scoping review

Study Population

- Adults undergoing abdominal surgery

\section{Comparators}

- Neuraxial anesthesia including spinal and epidural

- Placebo group

Search

- An initial search was conducted in PubMed, Scopus, and Cochrane libraries using the headings of "transversus

abdominis plane OR transverse abdominal plane OR TAP."

○ In the first search, "analgesia" was added.

- In a second search, "opioid OR opioids" was added.

- In a third search, "QI OR quality improvement" was added.

- A final search adding "evidenced based or EBP" was conducted, resulting in 10 articles that ranged from the years of 2012 to 2019 .

\section{Results}

- While all studies found some reduction in opioid use, the methods of evaluations and outcomes measured were different in many of the studies.

- The measurement of opioid use was inconsistent between studies.

Reduction in Opioid Consumption After TAP Block Amolong et al

Bacal et al

Baeriswyl et al

Brogi et al

흘 Felling et al

के

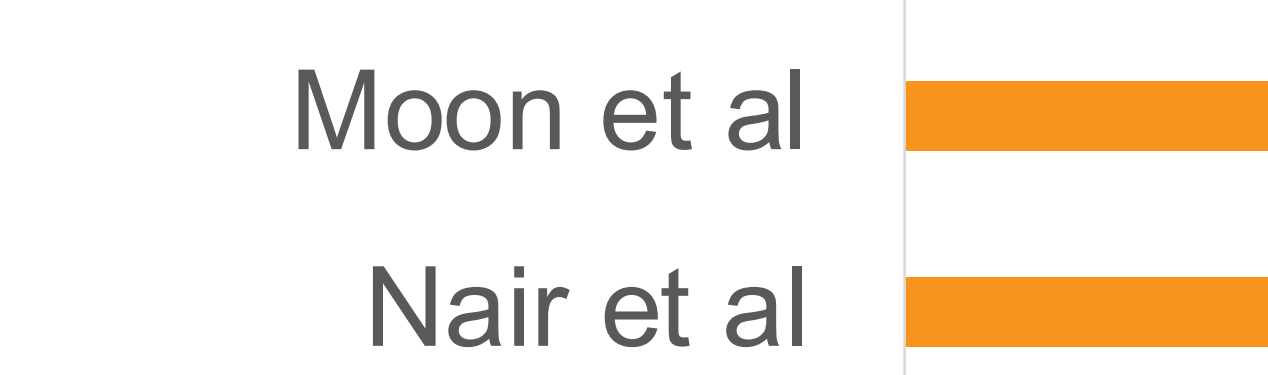

$$
\text { Nair et al }
$$

Theodoraki et al Tubog et al

$\begin{array}{lllrll}0 & 5 & 10 & 15 & 20 & 25 \\ & \text { Opioid Reduction }(\mathbf{m g}) & & \end{array}$

\section{Implications for Practice}

- Findings suggest that TAP blocks are an attractive option in a variety of abdominal surgeries.

- TAP blocks have been documented as a superior method of pain control with reduction in opioid consumption.

- The potential to reduce costs and decrease incidences of postoperative nausea and vomiting by utilizing TAP blocks in abdominal surgeries is another area for research.

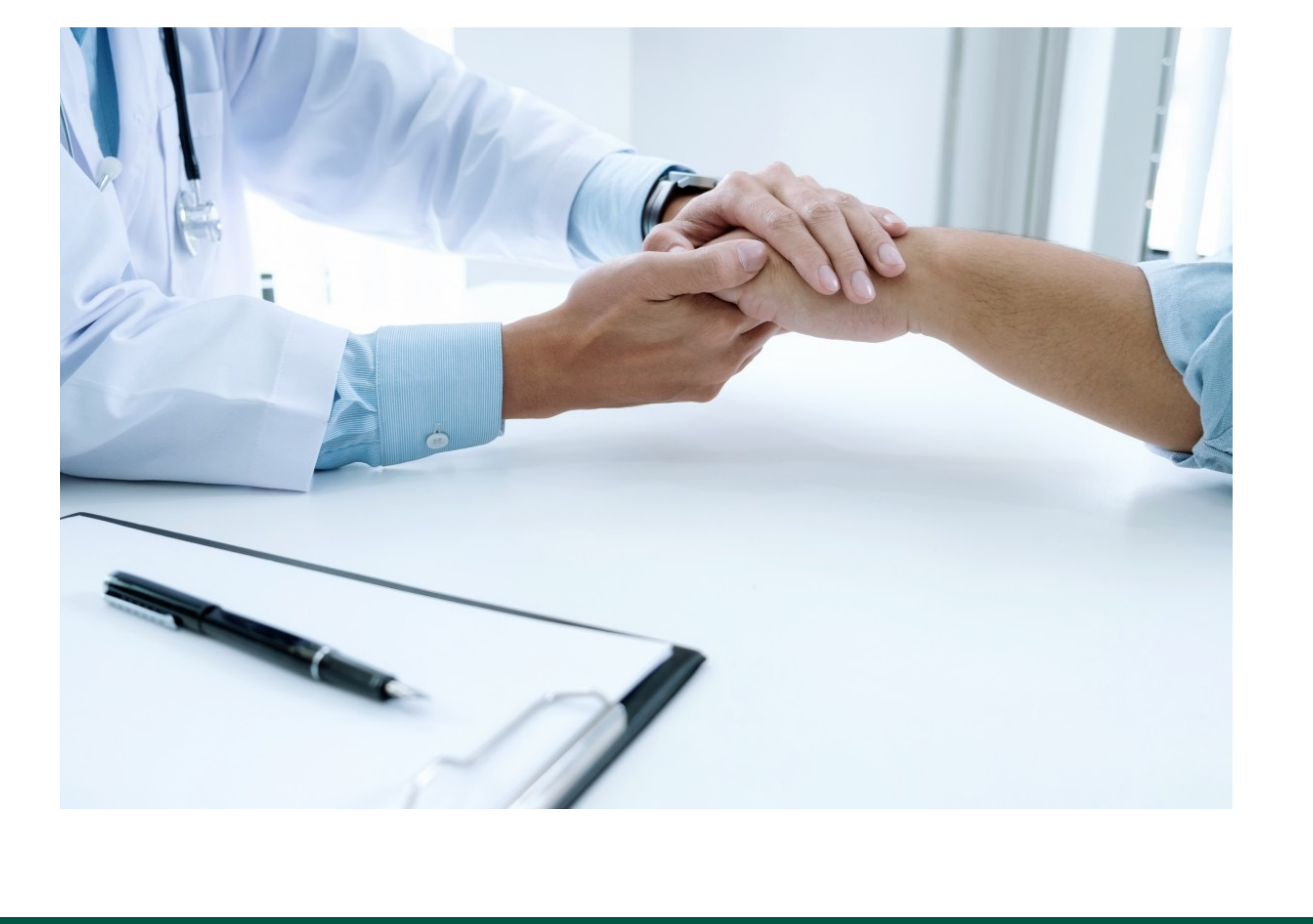

\section{References}

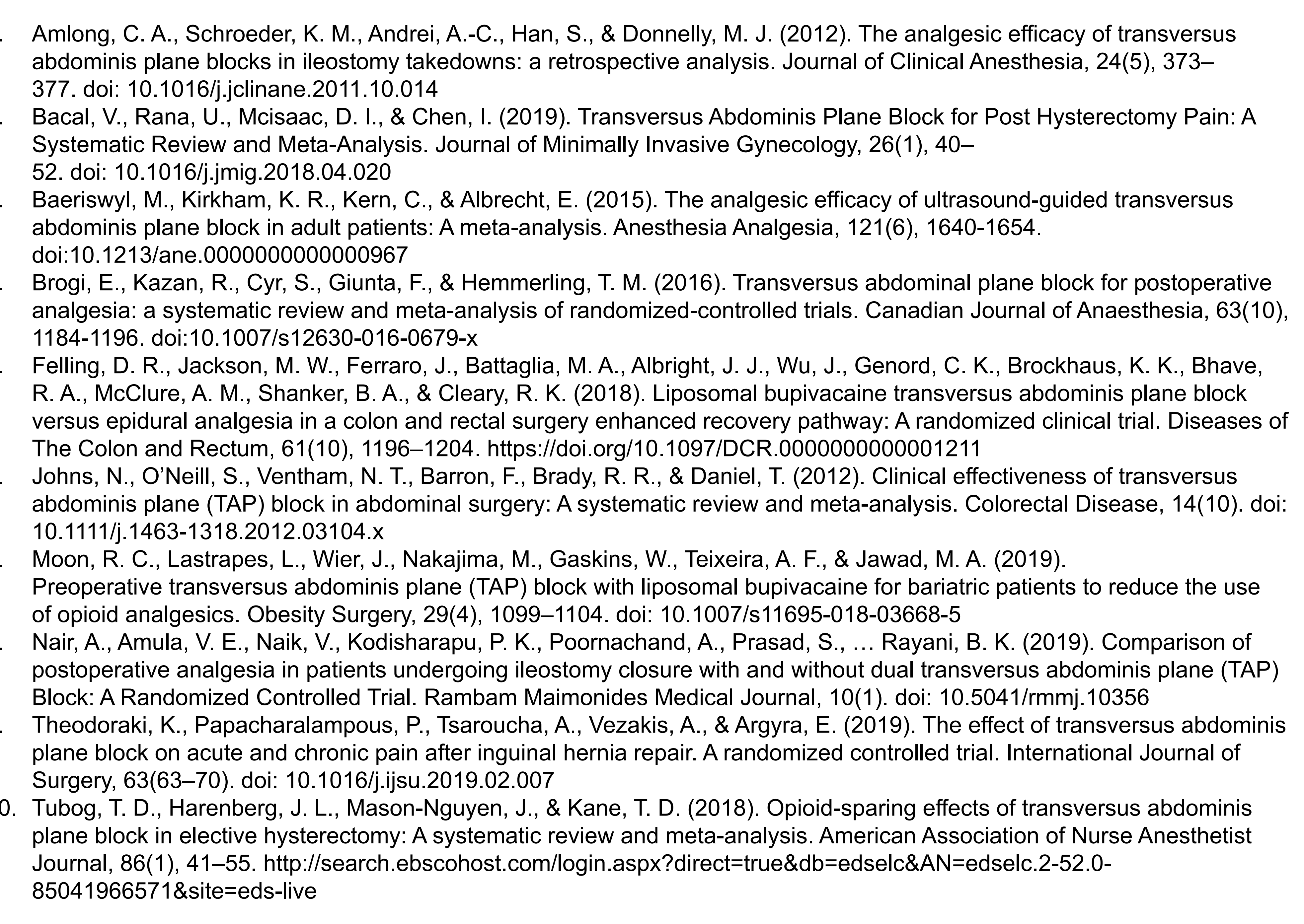

